\title{
ECOLEAD: A HOLISTIC APPROACH TO CREATION AND MANAGEMENT OF DYNAMIC VIRTUAL ORGANIZATIONS
}

\author{
Luis M. Camarinha-Matos ${ }^{1}$, Hamideh Afsarmanesh ${ }^{2}$, Martin Ollus ${ }^{3}$ \\ ${ }^{1}$ New University of Lisbon / Uninova, PORTUGAL, cam@uninova.pt \\ ${ }^{2}$ University of Amsterdam,THE NETHERLANDS, hamideh@science.uva.nl \\ ${ }^{3}$ VTT Industrial Systems, FINLAND, martin.ollus@vtt.fi
}

\begin{abstract}
The wide dissemination and effective materialization of the foreseen benefits of dynamic virtual organizations require a holistic approach to understand, model, and develop the needed infrastructures and tools to support the full life cycle of this organizational paradigm. Under this scope, the ECOLEAD integrated project was launched with the aim of creating the necessary foundations and mechanisms for establishing an advanced network-based industry society. The main underlying concepts, approach and preliminary results of this ongoing initiative are briefly summarized.
\end{abstract}

\section{INTRODUCTION}

The notion of dynamic virtual organizations (VO) has raised high expectations in various application domains. Among the potential benefits of these collaborative networks, a number of possibilities have been vastly mentioned in the literature, including increased access to market opportunities, sharing risks, reducing costs, achieving business goals not achievable by a single organization, etc. The rapid formation of a VO, triggered by a business opportunity and specifically tailored to the requirements of that opportunity, is also pointed out as an expression of agility, a survival element in turbulent market scenarios.

Pursuing these expectations, during the last $10 \sim 15$ years a large number of R\&D projects tried to establish technological foundations as well as operating practices for the support of Virtual Enterprises /Virtual Organizations. This effort is particularly visible in Europe through the European Commission funded programs (e.g. ESPRIT, IST, INCO), but also in the USA and other geographical regions (Australia, Brazil, Mexico, Japan, to name a few). Programs such as IMS (Intelligent Manufacturing Systems) also supported various projects in this area involving organizations from various continents.

This trend has so far led to an extensive amount of empirical base knowledge that now needs to be leveraged. In addition to the identification of many required components, tools, and the base infrastructure functionalities, awareness is being built and partially studied, even in the traditional collaborative organizations, regarding the fundamental configuration and operational rules, as well as the behavioral patterns that emerge. Nevertheless we are still far from a wide dissemination and adoption of this paradigm. It is now urgent to consolidate and 
synthesize the existing knowledge, setting a sound foundation for the future research and development in this area.

In fact, these initiatives, together with practical realizations of many variations of virtual organizations, have generated a large amount of empiric knowledge that is however still disperse and fragmented. The IST VOSTER project represented an attempt to consolidate some of this existing knowledge [6]. More recently, in part as a result of initiatives such as the THINKcreative [4] and VOmap [3,5] projects among others, it became evident that there is a need for investing on more fundamental research towards the creation of a sound theoretical foundation for VOs as well as a more holistic understanding of this paradigm. The ECOLEAD integrated project is an initiative in this direction which aims at creating the necessary foundations and mechanisms for establishing an advanced and networkbased industry society.

\section{ECOLEAD AND COLLABORATIVE NETWORKS}

A large variety of organizational forms of collaboration have emerged during the last years as a result of the many socio-economic challenges faced by society and enabled by the new ICT developments. Advanced and highly integrated supply chains, virtual enterprises, virtual organizations, professional virtual communities, value constellations, virtual institutes, and collaborative virtual laboratories, represent only the tip of a major trend in which enterprises and professionals seek complementarities and joint activities to allow them participate in competitive business opportunities, in new markets and / or reaching scientific excellence for innovative developments. Similar trends can be found in the none-profit / socialoriented contexts, e.g. in incident management, time bank, elderly care networks, etc.) [4]. All these examples are manifestations of the more general concept of collaborative network.

A collaborative network (CN) is constituted by a variety of entities (e.g. organizations and individuals) that are largely autonomous, geographically distributed, and heterogeneous in terms of their: operating environment, culture, social capital and goals. Nevertheless these entities collaborate to better achieve common or compatible goals, and their interactions are supported by computer networks. Unlike other networks, in $\mathrm{CN}$ collaboration is an intentional property that derives from the shared belief that together the network members can achieve goals that would not be possible or would have a higher cost if attempted by them individually.

Collaborative networks of organizations provide a basis for competitiveness, world-excellence, and agility in turbulent market conditions. They have the potential to support SMEs in identifying and exploiting new business potential, boosting innovation, and increasing their knowledge. Networking of SMEs with large-scale enterprises also contributes to the success of the big companies in the global market. Reinforcing the effectiveness of collaborative networks, mostly based on SMEs, and creating the necessary conditions for making them an endogenous reality in the European industrial landscape, are key survival factors.

A key question is however, how to guarantee the basic requirements to enable such dynamism and agility for collaborative networks. Among others, the formation 
of any collaborative coalition depends on some base commonality among its members, including: sharing of common or compatible goals, possessing some level of mutual trust, having established some common or interoperable computer infrastructures, and having agreed on some common policies, codes for practice and value systems, e.g. common policies for business practices in industry-based collaborative networks. Achieving these challenging base conditions is a prerequisite for agility in collaborative networks.

Related to the need for agility, another discussion point is whether collaborative networks shall be temporary or long-term establishments. While temporary organizations seem to better fit the dynamics of the market and the variable duration of business opportunities, long-term organizations better cope with trust building processes, and the investment on common infrastructures and code of practice. Some existing interesting and successful experiments have combined both types of organizations in a hybrid network, namely a long-term (growing and permanent) club or cluster of organizations that are willing and somewhat prepared to cooperate, and shorter-term coalitions involving subsets of these organizations that are dynamically assembled in order to respond to business opportunities. In this context, the ECOLEAD vision is that in ten years, in response to fast changing market conditions, most enterprises and specially the SMEs will be part of some sustainable collaborative networks that will act as breeding environments for the formation of dynamic virtual organizations.

The fundamental assumption in ECOLEAD is that a substantial increase in materializing networked collaborative business ecosystems requires a comprehensive holistic approach. Given the complexity of the area and the multiple inter-dependencies among the involved business entities, social actors, and technologic approaches, the substantial breakthroughs cannot be achieved with only incremental innovation in isolated areas. Therefore, ECOLEAD addresses three most fundamental and inter-related focus areas - constituting ECOLEAD pillars - as the basis for dynamic and sustainable networked organizations including: Breeding Environments, Dynamic Virtual Organizations, and Professional Virtual Communities.

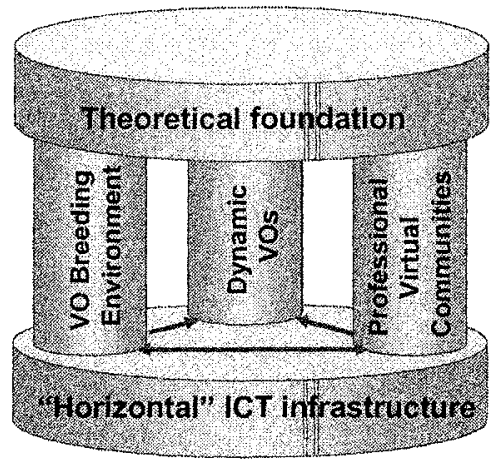

Figure 1 - ECOLEAD components
A VO Breeding Environment (VBE) represents an association or pool of organizations and their related supporting institutions that have both the potential and the interest to cooperate with each other, through the establishment of a "base" long-term cooperation agreement. When a business opportunity is identified by one member (acting as a broker), a subset of the VBE members can be rapidly selected to form a virtual organization. Long standing relationships regional clustering being an example - when driven by the willingness to cooperate and anchored on common business practices, form a natural business ecosystem where trust is incrementally built and where ad hoc collaborations can be established. Therefore VBE represents a group of organizational entities that have developed 
advanced preparedness for cooperation, for the cases when specific opportunities arise.

Dynamic Virtual Organization are temporary alliances of organizations that come together to share skills or core competencies and resources in order to better respond to business opportunities and produce value-added services and products, and whose cooperation is supported by computer networks. Within a VBE context the $\mathrm{VO}$ is rapidly assembled into a business entity enabling the actual collaboration of enterprises and individuals to respond to a specific collaboration opportunity (business oriented or other). The temporary nature of VOs, the needed interorganizational processes, and the potentially diverging objectives of the partners, require the development of a VO management system, which is based on the preparedness created in the VBE and covering the full life cycle of the VO, namely its creation, operation, and dissolution phases.

Professional Virtual Community (PVC) represents the combination of concepts of virtual community and professional community. Virtual communities are defined as social systems of networks of individuals, who use computer technologies to mediate their relationships. Professional communities provide environments for professionals to share the body of knowledge of their professions such as similar working cultures, problem perceptions, problem-solving techniques, professional values, and professional behavior. PVCs cannot be dissociated from the underlying business ecosystem of the society, due to their contractual links (socialbounds) with all the consequences at the intellectual property and life maintenance levels. PVCs are one of the most relevant elements for keeping the business ecosystem "alive" and for launching and operating dynamic VOs of the future.

The ECOLEAD pillars are supported and reinforced by two horizontal developments:

Theoretical foundation. Sustainable development of collaborative networked organizations needs to be supported by strong fundamental research leading to the establishment of Collaborative Networks as a new scientific discipline. Ad-hoc approaches and poor understanding of the behavior of the collaborative structures and processes mainly characterize the past developments in the area of collaborative networked organizations. There is not even a commonly agreed definition for the virtual organization concept. ECOLEAD includes the establishment of a sound theoretical foundation, and a reference architecture at all levels, as a pre-condition for the next generation of collaborative networks.

Horizontal ICT infrastructure. Implantation of any form of collaborative network depends on the existence of an ICT infrastructure. In order to leverage the potential benefits of the collaborative networked organization paradigm, more flexible and generic infrastructures need to be designed and implemented. The lack of common reference architectures and generic interoperable infrastructures, together with the rapid evolution of the underlying technologies, represents a major obstacle to the practical evolution of the area. As part of the ECOLEAD foundation, a generic, transparent, easy to use and affordable horizontal infrastructure for collaboration is being designed. 


\section{VO BREEDING ENVIRONMENTS}

\subsection{Aims}

Traditionally, clusters/associations of organizations are established in a geographic region [1], with the advantage of having common business culture, and sense of community, and typically focused on one of the specialty sectors of the region. What is challenging today is to tackle this restriction, and at best replace it by a new "support-environment" called a breeding environment, that applies effective Information/communication infrastructures to provide common grounds for interaction/collaboration, facilitates the configuration and establishment of VOs, assists with the operation of VOs, introduces new approaches and mechanisms to build trust, defines a collaboration business culture, and the common value systems and working/sharing principles among independent organizations, even from different geographical regions. Cultural ties and particular human relationships are important motivating factors to start up and form such associations representing the VBE, as the support environment for dynamic formation of VOs.

VBE is a regulated open but controlled-border association of its members. It aims at improving the preparedness of its member organizations for joining the potential future VOs, hence providing a cradle for dynamic and agile establishment of opportunity-driven collaborative networks. Proper management of the VBE during its entire life cycle is at the heart of this support environment. Some of the main aims of the VBE can be formulated as follows:

- Establish the base trust for organizations to collaborate in VOs, by gathering/preparing the credibility records of organizations, as well as the definition of proper credit-assignment principles.

- Reduce the cost/time to find suitable partners for configuration of the VOs.

- Assist with the creation, reaching agreements, and contract negotiation for establishment of VOs.

- Assist with the dynamic re-configuration of the VOs, thus reducing the risk of big losses due to some organization failures.

- Provide some commonality for interaction and "accepted business culture", by offering:

- Base ICT infrastructure (for collaboration), thus reducing the set up times during the VO formation.

- Cooperative business rules (measured by the developed common metrics to evaluate member's credibility \& performance).

- Template contracts for involvement in VOs (samples are provided for VOs).

- Base ontology for the sector (to be incrementally developed within the VBE). VBE can also serve as the basis for some support institutions (e.g. insurance companies, education organizations, etc.) to provide the so called "life maintenance" facilities to its members.

\subsection{Actors and roles in a VBE}

Organizations or actors in a VBE can include:

- Business entities providing products and services to the market that get involved in the VOs to gain quantitative profit.

- Non-profit institutions that get involved in the VOs to gain qualitative profit. 
- VO Support institutions, for example: legal and contractual service providers, companies supporting life maintenance to individuals (e.g. insurance and training companies), ministries, sector associations, chamber of commerce, environmental organizations, etc.

Participants in a VBE can play several roles. The following main roles are considered:

- VBE member - this is the basic role played by those organizations that are registered at the VBE and are ready to participate in the VBE activities.

- VBE administrator - performed by the organization responsible for the VBE operation and evolution, promotion of cooperation among the VBE members, filling the skill/competency gaps in the VBE by searching and recruiting/inviting new organizations into the VBE, daily management of the VBE general processes, e.g. assignment/reassignment of rights to different actors in the VBE based in their responsibilities, conflict resolution, preparation of bag of assets, making common VBE policies, among others.

- Opportunity Broker or simply Broker - performed by a VBE actor (a VBE member organization or an individual representing a VBE member) that identifies and acquires new collaboration opportunities (business opportunities or others), by marketing VBE competencies and assets and negotiating with (potential) customers. There is the also the possibility of this brokerage function being played by an outside entity as a service to the VBE.

- VO Planner or business integrator - performed by a VBE actor that, in face of a new collaboration opportunity (designed by an opportunity broker), identifies the necessary competencies and capacities, selects an appropriate set of partners (VBE members and even outsiders in case there is not enough competencies and/or capacities inside the VBE), and structures the new VO. In many cases the roles of opportunity Broker and VO planer are performed by the same actor.

- VO coordinator - performed by a VBE actor that will coordinate a VO during its life cycle in order to fulfill the goals set for the collaboration opportunity that triggered the VO.

Additional roles that might be useful considering in a VBE are: VBE advisor (or an advisory board), VBE Service provider, VBE Ontology provider, etc.

In general it is possible that several roles are performed by the same actor. The access rights to the information and support tools available in the VBE management system will depend on the specific role played by an actor.

\subsection{VBE life cycle}

The life cycle of the VBE represents all the stages that a VBE may go through, from its creation stage, to its operation, and possible dissolution. In fact VBE, being a long-term alliance, and considering its valuable bag of assets gradually collected in the VBE, its dissolution is a very unusual situation. Instead, it is much more probable that the VBE goes through another stage, a so called metamorphosis stage, where it can evolve and change its form and purpose. On the other hand, it is the case that only during the operation stage of the VBE, the VO can be created. VBEs by nature are self organizing and can be modeled and represented following the 
principles of the Chaordic system thinking [7]. Fig.2 represents the five stages of the VBE life cycles in a chaordic diagram.

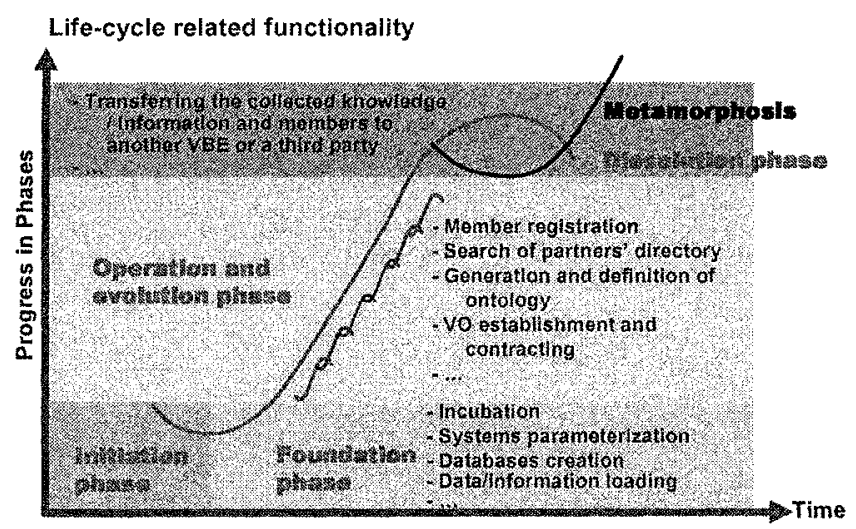

Figure 2 - VBE life cycle in terms of chaordic systems thinking

Considering that typically VBEs serve specific sectors/domain and have specific aims, there will be many different sector/domain-dependent VBEs needed to be established in future in order to support the creation of different forthcoming VOs. Therefore, it is important to provide support for all stages of the VBE's life cycle and not only focus on its operation stage.

\subsection{VBE Management}

Management of the VBE during its life cycle is at the heart of the VBE research and development. Due to the fact that there are so far no defined "reference models" for the VBEs, to address their different aspects including their behavior, structure, topology, cultural/legal framework, etc., there are no clear definitions of what exact activities are associated with the VBEs, that need to be supported by their management system. However, several examples of the traditional industry-based VBEs can already be found in practice that can be used as a source of inspiration for our work, e.g. the cases represented by Virtuelle Fabrik [10] and ITESM [8,9]. Defining a comprehensive and generic "reference model" for VBEs very challenging. Nevertheless, with basis on the initial empiric knowledge that can be gathered from existing cases, it is realistic to gradually define a "reference framework for VBEs", addressing some of the aspects of the reference model for VBEs, such as its behavior, topology, and structure.

Furthermore, simultaneous to the definition of this reference framework, our approach is to design a system architecture for the VBEs management, defining models of its components as well as methodologies and mechanisms to support its behavior. Earlier studies performed by several members of the ECOLEAD consortium in some other VO related projects, including: THINKcreative [4], VOmap [5], VOSTER [6], PRODNET [2], etc., are used as the starting point. So far, with the initial studies in ECOLEAD, the following general base required functionality are identified for different stages of the VBE life cycle:

Base functionality supporting the VBE creation - This phase includes two main steps: (1) initiation / recruiting, which requires the establishment and setup of a 
common base infrastructure, recruiting potential organizations to join the VB, and establish some base ontology / thesaurus of the domain, to establish the vision and strategic objectives of the VBE are defined; (2) VBE foundation, requiring support for parameterization of the used systems, setting up the necessary links, creation of the necessary databases (with initial meta-data / ontology), and populating these information structures.

Base functionality supporting the VBE operation and evolution - This phase requires support for: (i) Management of competencies and assets, (ii) Registration of new members (including profiling, characterization of competencies, products, services, etc.), (iii) Assisting VO creation, (iv) Incremental generation / evolution of meta-data / ontologies for the domain / sector, (v) Keeping records of past performance and collaboration processes, (vi) Assessment and assistance tools, (vii) Collaboration support (e.g. newsgroups, discussion forum, common information repositories, etc.), (viii) Management and evolution of working and sharing principles and rules, (ix) Acquisition and management of common knowledge and assets.

Base functionality supporting the VBE metamorphosis - This phase will require assistance for the design of the aimed new organizational structure, selection and reorganization of the information and knowledge collected during the VBE operation and that might be transferred to the new organization, analysis and adjustment to the new context, etc. In the case of $V B E$ dissolution there is a need to plan the transfer of its collected knowledge, information, bag of assets to its members or another organization based on defined agreements.

\section{VO CREATION}

\subsection{VO creation and VBE}

One important issue in collaborative networks is the formation of dynamic VOs/VEs. How can we quickly plan, find partners, and organize them in a collaborative network once a business or collaboration opportunity shows up?

Early works assumed that partners could be easily selected from the wide open universe of available enterprises / organizations. This assumption however ignores a number of difficulties:

- How to know about the mere existence of potential partners in the open universe and deal with incompatible sources of information?

- How to acquire basic profile information about organizations, when there is no common template or standard format?

- How to quickly establish an inter-operable collaboration infrastructure, given the heterogeneity of organizations at multi-levels, and the diversity of their information systems?

- How to build trust among organizations, which is the base for any collaboration?

- How to develop and agree on the common principles of working and sharing?

- How to quickly define the agreements on the role and responsibilities of each partner, to reflect sharing of tasks and the rights on the produced results?

Perhaps influenced by the developments in the e-commerce area, lately some authors started to consider markets of enterprises / organizations as a source for potential partners. The notion of market would provide means to find organizations 
(market directory) and perhaps some normalized profile and even some (minimal) references about those organizations. This is a concept frequently adopted by simple multi-agent based systems for partners' selection.

However, when the goal is to find partners for collaboration, the notion of market does not satisfy all necessary features. In order to support rapid formation of consortia it is necessary that potential partners are ready to participate in a collaborative network. This readiness includes common (interoperable) infrastructure, common operating rules, cooperation agreement, etc. Collaboration also requires a level of trust among organizations that is not a typical requirement in a simple market. Therefore, ECOLEAD considers the existence of a breeding environment [2] as a necessary context for the effective creation of virtual organizations. VO creation is the first step of the VO life cycle with the VBE operation phase (Fig. 3).

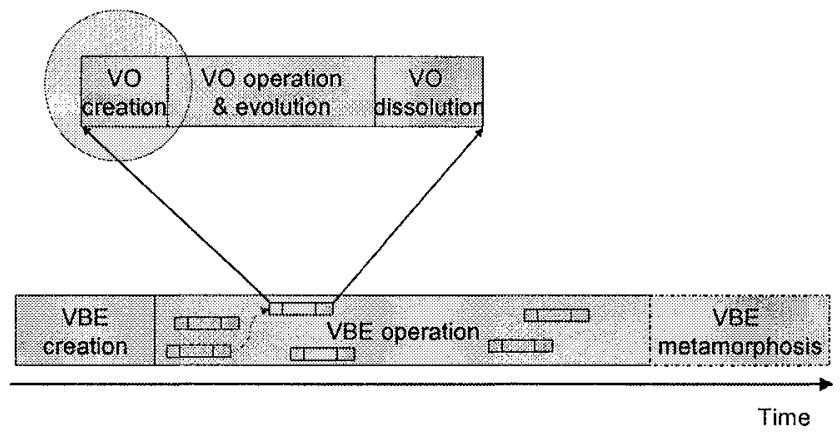

Figure 3 - VO creation within the life cycle of a VO and a VBE

\subsection{VO creation process}

The following main steps (Fig. 4) have been identified for the VO creation process:

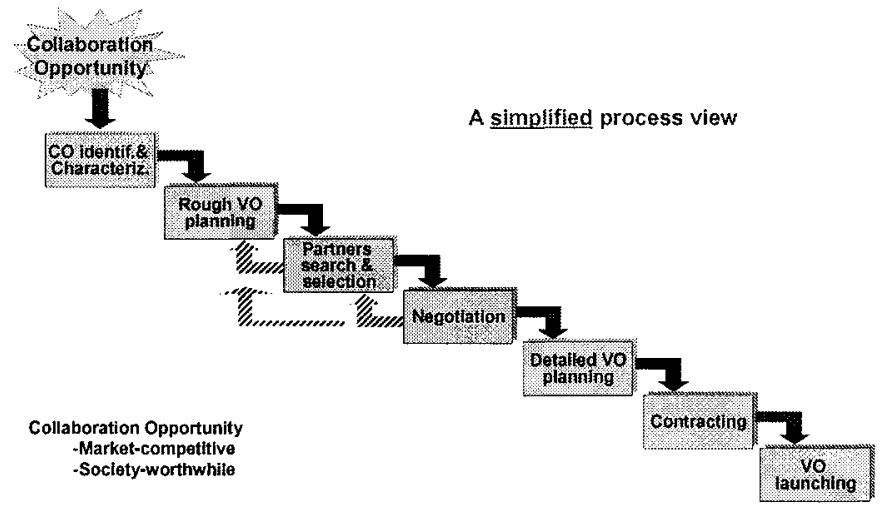

Figure 4 - Simplified VO creation process

- Collaboration Opportunity Characterization: this step involves the identification and characterization of a new Collaboration Opportunity $(\mathrm{CO})$ that will trigger the formation of a new VO. Collaboration opportunities might be external, originated by a (potential) customer and detected by a VBE member acting as a broker. Some opportunities might also be generated internally, as part of the development strategy 
of the VBE. Support tools for CO representation and feasibility analysis are important at this stage. The main actors involved in this step are the broker and the customer).

- Draft VO planning: determination of a rough structure of the potential VO, identifying the required competencies and capacities, as well as the organizational form of the VO and corresponding roles. Functionalities to support the structural and topological design of the VO architecture, model the (macro) collaborative process, and assess (simulation) different options are necessary. The main roles involved in this step are the broker and the VO planner.

- Partners search and selection: perhaps one of the most addressed topics in past research, this step is devoted to the identification of potential partners, and their assessment and selection. Search mechanisms, assessment criteria, analysis of past performance record and level of preparedness, etc., are among the necessary support functions. This phase requires mainly the VO planer and VBE member roles.

Negotiation: is an iterative process to reach agreements and align needs with offers. It can be seen as complementary to the partners' selection process. Negotiation protocols and mechanisms, decision making process, and forms of representation of agreements are important requirements here. The VO planner and VBE member are the main roles involved here.

Detailed VO planning: once partners have been selected and collaboration agreements are reached, this step addresses the refinement of the VO plan and its governance principles. Assignment of roles and responsibilities, definition of operating rules and further refinement of the (macro) collaboration process are included in this phase. The VO planner and VBE member are the main roles required in this step. Interactions with the broker might also be necessary.

- Contracting: involves modeling of contracts and agreements as well as the contracting process itself, before the VO can effectively be launched. Semiautomatic or even automatic mechanisms can be considered for some application domains. This step might be considered in close interaction with the negotiation phase. Main roles involved in this step are the VO planner, the VBE member, the VO coordinator and possibly the broker and the customer.

- VO launching: the last phase of the VO creation process, i.e. putting the VO into operation, is responsible for tasks such as configuration of the ICT infrastructure, instantiation of the collaboration spaces, assignment and set up of resources, notification of the involved members, and manifestation of the new VO in the VBE. Main roles required here are the VO coordinator, the VBE member, and possibly the VBE administrator.

\section{VO MANAGEMENT}

The definitions of a VO often assume that a virtual organization behaves and can be managed in some way like a single organization. However, major challenges for VO management come from two characteristics: the temporary nature of a VO and the distribution of operations in independent but interdependent organizations with their own aim, behavior and culture. 
To achieve an efficient management of VOs, ECOLEAD takes a broad approach towards VO Management by defining that VO Management denotes the organization, allocation and co-ordination of resources and their activities as well as their inter-organizational dependencies to achieve the objectives of the VO within the required time, costs and quality frame.

VO management applies knowledge, skills and/or tools in order to achieve the VO goals. The management of Virtual Organizations to a large extent deals with humans and it is performed by humans. In most cases the human aspect is considerable as the last decisions about management actions usually are done by the VO managers, who also may imply different management styles. However, it is not always possible to rely on management experience and thus systematic means and tools are needed.

The required dynamic management implies that needs of management actions are identified in real time. Consequently, an efficient performance measurement system should also be in place to give reliable, real-time indicators about the performance of the VO. The basic challenge is to develop real time-time performance measurement based management approaches fulfilling the requirements and features of the definition above. The behavior of such a management approach is illustrated in Fig. 5, where the management is considered as a real-time control loop.



Figure 5-VO management illustrated as a control loop

The efficiency of a VO can be judged from three different viewpoints:

1.The fulfillment of the task, i.e. keeping expected costs, time and quality

2.The efficiency of the VO and the collaboration

3. The efficiency of the management approach and management methods

Most of the VO management actions are devoted to ensure an efficient task fulfillment. The indicators for getting this performance are also mostly related to obvious measurements, mentioned above. Actions to maintain or enhance this performance are usually devoted to coordination of activities among the partners in the VO taking into account the challenges coming from the features of the VO.

The efficiency of virtual organizations depends heavily on the performance of the partners and their collaboration. This performance may, in addition to the task, depend on the configuration of the VO. The relationship between partner performance and the task fulfillment is not easy to model, nor are there obvious 
measurements available. Some of them are also qualitative and perhaps even subjective. However, the virtual breeding environment (VBE) would benefit from information about some indicators or measurements. Such information is essential in the creation of new VOs and is assumed to be collected as parameters in the value system of the VBE. One of the challenges in is to create models for judging and managing partners' performance based on the concrete measurements available and the processes established for the task fulfillment.

The aim of the work on VO Management is to develop efficient approaches for this Management. Consequently, also the efficiency of the management itself needs to be understood. Issues like task distribution, coordination principles, incentive systems, information flows, etc have an impact on the performance of a VO. Like for the partner performance, the challenge here is to develop a better understanding of the relationship between management approach and the fulfillment of the tasks given to the VO. This relationship should give the basis for the measurement of the management performance and also support possible actions for enhancement of the management.

The approach requires an appropriate monitoring and measurement system. Consequently, the development of a performance measurement system supporting the VO Management is a key activity. This system has to rely on models of the processes to be managed in order to help in identifying the relevant management actions in different situation. The aim of the ECOLEAD project is to integrate the performance measurement and VO Management and demonstrate the performance based VO Management approach.

In the dissolution phase of a VO, all valuable outcome from the VO need to be handled in a proper way and given to sustainable entities within the VBE (and PVC if individuals from a PVC are involved). In addition to the outcome of the task fulfillment, which can contain product documents, warranties, IPR rights, etc, the VBE may need information about experiences and the performance of the VO to be considered in the creation of future VOs. Also knowledge about customers and markets, collected during the VO operation, are important in future activities. The experience may be used by future VOs. In order to support such a support such actions, ECOLEAD will focus also on the management of the inheritance of the achievements of VOs.

Based on the aimed developments described above, a collection of e-support functions for the management of VOs are being designed and developed.

\section{INTERACTIONS WITH PVC}

Part of the ECOLEAD holistic approach includes the investigation of the interrelationships between the VBE/VO and the PVC. In fact the importance of the "human component" in the formation and promotion of new organizational forms is becoming a more relevant research issue.

Analogy is one of the perspectives to exploit. A PVC can be seen as the counterpart of a VBE when the focus is on collaborative networks of professionals. A Virtual Team (VT) is analogous to a VO in the sense that it is a temporary group of people, members of the PVC that get engaged in jointly taking over a business opportunity. Therefore, at a conceptual level, most logical components of the 
VBE/VO framework can apply to PVC/VT. Some issues, however, require a different focus in the case of PVC/VT. That is, for instance, the "life maintenance institutions" that seem fundamental for a sustainable PVC, or some support services (e.g. life long training) addressing the needs of humans.

Another perspective is related to the interactions between the two classes of organizations when they co-exist in the same business ecosystem. PVC members might be members (employees) of organizations involved in a VBE. This can happen either when the VBE promotes the existence of the PVC or when the PVC exploits additional skills that are not relevant to the core business of the employers. In the first case the VBE might use the PVC as a mechanism to leverage its participation in dynamic markets and boosting innovation, by creating new motivation to their members. In the second case, the competencies exploited by the PVC might be outside the contractual bounds between professionals and their employers, but nevertheless contribute to generate new business opportunities with benefits for all.

It is therefore important to better understand:

- The role of PVCs in launching / triggering new VOs;

- The formation of hybrid organizations VO-VT;

- The limits and opportunities of the contractual bounds between PVC members and their counterparts in the VBE;

- The possibilities of VTs formed to solve problems of the VBE, VO, or a single VBE member.

These are important research issues requiring further work in order to design appropriate support tools and policies.

\section{CONCLUSIONS}

Collaborative networks are already recognized in the society as a very important instrument for survival of organizations in a period of turbulent socio-economic changes. A growing number of collaborative-networked organization forms are emerging as a result of the advances in the information and communication technologies, the market and societal needs, and the progress achieved in a large number of international projects.

Nevertheless most of the past initiatives have addressed only partial aspects, failing to understand and properly support the various business entities and their inter-relationships in complex and fast evolving business ecosystems. The ECOLEAD project is pursuing a more holistic approach considering both long-term and temporary organizations as well as networks of organizations and networks of people. A new framework for advance collaborative networked organizations is expected from this integrated project.

Complementarily the ECOLEAD project is also addressing the need for establishing a more sound theoretical foundation for collaborative networks in order to turn this growing research area into a recognized new scientific discipline.

This paper introduced the approach and current developments towards the stated goals. 
Acknowledgments. This work was supported in part by the ECOLEAD project funded by the European Commission. The authors thank the valuable contributions of their partners in the ECOLEAD consortium.

\section{REFERENCES}

1. Bremer, C.; Mundim, A.; Michilini, F.; Siqueira, J.; Ortega, L. - A Brazilian case of VE coordination, in Infrastructures for Virtual Enterprises, Kluwer Academic Publishers, Boston, Oct 1999.

2. Camarinha-Matos, L.M.; Afsarmanesh, H. - Elements of a base VE infrastructure, L.M. Camarinha-Matos, H. Afsarmanesh, J. Computers in Industry, Vol. 51, Issue 2, Jun 2003, pp. 139-163.

3. Camarinha-Matos, L.M.; Afsarmanesh, H. - A roadmap for strategic research on virtual organizations. In Processes and Foundations for Virtual Organizations, Kluwer Academic Publishers, Boston, 2003.

4. Camarinha-Matos, L.M.; Afsarmanesh, H. (Ed.s) - Collaborative networked organizations $-A$ research agenda for emerging business models, Kluwer Academic Publishers, Boston, 2004.

5. Camarinha-Matos, L.M.; Afsarmanesh, H.; Loeh, H.; Sturm, F.; Ollus, M. - A strategic roadmap for advanced virtual organizations. In Collaborative Networked Organizations: A research agenda for emerging business models, Kluwer Academic Publishers, Boston, 2004.

6. Camarinha-Matos, L. M.; Afsarmanesh, H., Ollus, M. - Virtual Organizations: Systems and Practices, Springer, Boston, 2005.

7. van Eijnatten, F. M. - Chaordic systems thinking chaos and complexity to explain human performance management, in Proceedings of Business Excellence I (G. Putnik, A. Gunasekaran, Ed.s), ISBN 972-8692-08-0, University of Minho, Portugal, 2003.

8. Mejia, R.; Molina, A. - Virtual enterprise broker: Processes, methods and tools, in Collaborative business ecosystems and virtual enterprises, Kluwer Academic Publishers, Boston, May 2002.

9. Molina, A. and Flores M. - Exploitation of Business Opportunities: The role of the virtual enterprise broker, in E-Business and Virtual Enterprises. Managing Business to Business Cooperation (Camarinha-Matos, Afsarmanesh and Rabelo, Ed.s), Kluwer Academic Publishers, Boston, Dec. 2000.

10. Plüss, A.; Huber, C. - Virtuellefabric.CH - A source network for VE in mechatronics, in Virtual Organizations - Systems and Practices, Springer, Boston, 2005. 